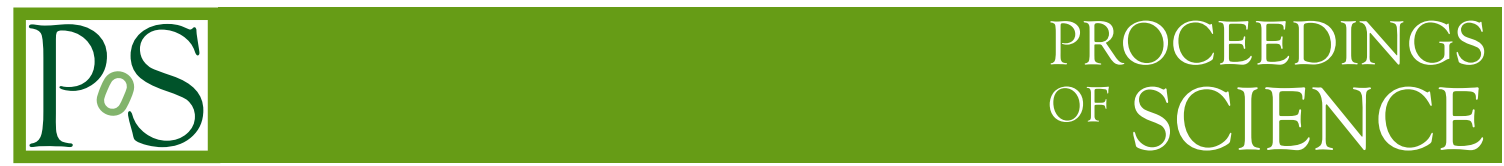

\title{
A brief (p)review on a possible fourth generation world to come
}

George W.S. Hou* ${ }^{\dagger}$

Department of Physics, National Taiwan University, Taipei, Taiwan 10617

E-mail: wshoulphys.ntu.edu.tw

35th International Conference of High Energy Physics - ICHEP2010,

July 22-28, 2010

Paris France

\footnotetext{
*Speaker.

${ }^{\dagger}$ Supported by National Science Council and National Center for Theoretical Sciences.
} 
The $t^{\prime}$ and $b^{\prime}$ quarks enjoy nondecoupling in the $Z$-penguin and box diagrams [1,2], bringing in a new $C P$ violating (CPV) phase through $V_{t^{\prime} s}^{*} V_{t^{\prime} b}[3]$ into $b \rightarrow s$ transitions. For those who still cite neutrino counting or electroweak precision tests, please see the "Four Statements" [4, 5].

In the following, we pick up the thread of $B \rightarrow K \pi$ direct CPV (DCPV) difference, linking to the 4th generation prediction (and current quest) of $t$-dependent CPV (TCPV) in $B_{s} \rightarrow J / \psi \phi$. We then soar up to the heavens with possible source of CPV for the Universe (CPV-4-U); for the "cauldron" that stirs strong phase transitions, we raise the possible link between electroweak symmetry breaking and large Yukawa couplings (4-EWSB). With the prognosis for 2011, 2012 and beyond, we stress these as preview for the possible 4th generation to come: CPV-4-U and the raison d'être for our Universe (and ourselves); 4-EWSB and the raison d'être for the LHC itself.

\section{Twilight: $B \rightarrow K \pi$ DCPV Difference}

Between BaBar and Belle, DCPV was observed in 2004: $A_{K^{+} \pi^{-}} \cong-10 \%$. Though not significant in itself, $A_{K^{+}} \pi^{0}$ deviated from $A_{K^{+} \pi^{-}}$by $3.8 \sigma$, which was not anticipated. In 2008, Belle data alone showed a difference [6] of $4.4 \sigma: \Delta A_{K \pi} \cong 16 \%>\left|A_{K^{+} \pi^{-}}\right|$is strikingly large!

Struck by the strength of the difference, we utilized nondecoupling and the new complex factor of $V_{t^{\prime} s}^{*} V_{t^{\prime} b}$ and demonstrated [7], using PQCD factorization at LO (equivalent to QCDF at NLO), that the $t^{\prime}$ quark can in principle generate $\Delta A_{K \pi}$. The work was further refined [8] at NLO in PQCD, where having some "color-suppressed tree" amplitude $C$ made it easier to account for $\Delta A_{K \pi}$. As the $b \rightarrow s Z$-penguin and $b \bar{s} \leftrightarrow \bar{b} s$ box diagram are cousins, we predicted [7] $\sin 2 \Phi_{B_{s}}$ (CPV phase of the said box amplitude) to be in the range of -0.2 to -0.7 . It was further illustrated [9] how the complete $4 \times 4$ CKM matrix could be determined, by facing all flavor constraints.

Boxes and $Z$-penguins have provided us with a wealth of information on flavor physics and CPV. From the $K^{0}-\bar{K}^{0}$ box, we learned the GIM mechanism and existence of charm, and accounted for $\varepsilon_{K}$; we inferred heavy top from the $B_{d}$ box, and eventually measured $\sin 2 \phi_{1} / \beta$. With heavy top, the $s \rightarrow d Z$-penguin diminished the value for $\varepsilon^{\prime} / \varepsilon$ and gave the value for $K \rightarrow \pi v \bar{v}$, giving $Z$-penguin dominance of $b \rightarrow s \ell^{+} \ell^{-}$and the measurable top effect in $Z \rightarrow b \bar{b}$. All that, with 3 generations. Just wait if there exists a 4 th generation - it will bring out an agenda for all aspects of flavor physics and CPV: $B_{s}, A_{\mathrm{FB}}\left(B \rightarrow K^{*} \ell^{+} \ell^{-}\right)$, renewed interest in $Z \rightarrow b \bar{b}, \varepsilon_{K}, \sin 2 \phi_{1} / \beta$, and to a lesser extent, $\varepsilon^{\prime} / \varepsilon$ and $D$ mixing. But as we shall see, the most exciting aspect would be the direct search for $t^{\prime}$ and $b^{\prime}$ quark at the LHC, with continued interest at the Tevatron.

\section{PAMELA!}

But let me bring myself back to how things were before 2008, by drawing analogy with "PAMELA". The excess of energetic $e^{+}$observed by PAMELA and others could in fact be due to nearby pulsars [10], hence astrophysical. BUT THAT DIDN'T STOP THE DM (Dark Matter) PARTICLE SPECULATOR/THEORISTS. In contrast, as alluded to, the $B \rightarrow K \pi$ DCPV difference could also arise from "enhanced color-suppressed $C$ ", AND THIS SEEMS TO STOP FURTHER THOUGHTS ACROSS (the) ATLANTIC!? The possibility of new physics in $b \rightarrow s Z$-penguin, and the prediction for large CPV effect in $B_{s} \rightarrow J / \psi \phi$, was largely ignored during 2005-2007. We remark that QCDF did not predict $A_{K^{+} \pi^{-}}$, while SCET got the sign for $\Delta A_{K \pi}$ totally wrong, after taking $A_{K^{+} \pi^{-}}$as input. It was PQCD which anticipated, ca. 2001, the sign as well as strength of $A_{K^{+} \pi^{-}}$. As much as experimentalists do "blind analysis", theorists should make "predictions". 


\section{Moonshine: Prediction/Quest for TCPV in $B_{s} \rightarrow J / \psi \phi$}

In the CDF public note CDF/ANAL/BOTTOM/PUBLIC/9458, it is stated "George Hou predicted the presence of a $t^{\prime}$ quark ... to explain the Belle result and predicted a priori the observation of a large $C P$-violating phase in $B_{s} \rightarrow J / \psi \phi$ decays [7, 8]". "Ref. [7]" is our Ref. [7], while "Ref. [8]" is a refinement [11] of $\sin 2 \Phi_{B_{s}}$ prediction to the range of -0.5 to -0.7 (SM value is $\sim-0.04$ ). Surprisingly, three measurements, by CDF in $12 / 2007$ and $8 / 2008$ and D $\emptyset$ in $2 / 2008$, gave central values right in this range!! The error was, of course, large: despite some rattling by UT fit, and taking a year to combine the two experiments, the significance was only $2.1 \sigma$ [12] in summer 2009. Note that CDF uses $-\sin 2 \beta_{s}$ while D $\emptyset$ uses $\sin \phi_{s}$ instead of our $\sin 2 \Phi_{B_{s}}$.

Summer 2010 was volatile. First, $\mathrm{D} \emptyset$ splashed forth a value of $A_{\mathrm{SL}}$ that deviated by $3.2 \sigma$ from SM expectations. ${ }^{1}$ A week later, CDF uncovered their new $\sin 2 \beta_{s}$ value, which dropped to only $\sim 1 \sigma$ away from SM. Even though $\mathrm{D} \emptyset$ released a larger new value for $\sin \phi_{s}$, the verdict seems to be that $\sin 2 \Phi_{B_{s}} \equiv \sin \phi_{s} \equiv-\sin 2 \beta_{s}$ is weaker in strength than in 2009. Interestingly, we had just studied the case for heavier $m_{t^{\prime}}=500 \mathrm{GeV}$ (rather than $300 \mathrm{GeV}$ in earlier studies), turning out a central value of $\sin 2 \Phi_{B_{s}} \sim-0.33$ [14]. A heavier $t^{\prime}$ seems preferred now by CPV data.

The sad thing for Tevatron is, with a target central value of -0.3 rather than -0.6 , there is little hope that the combined Tevatron result would ever reach "evidence" level, given that CDF and $\mathrm{D} \emptyset$ results are already using datasets of order $5-6 \mathrm{fb}^{-1}$. But it is assured that the value should be quickly measured by LHCb, once it has even a couple of $100 \mathrm{pb}^{-1}$, which could arrive before summer 2011. Another thing worthy of note is that the 4th generation seems "rehabilitated" since start of 2010, judging from significant work even just on flavor and CPV physics [15].

\section{Starry Heavens: CPV 4 Universe ?}

Recall the Sakharov conditions for generating Baryon Asymmetry of the Universe (BAU): Baryon Number Violation; CP Violation; Out of Equilibrium. In his Nobel Lecture, Kobayashi admitted that "Matter dominance of the Universe seems requiring new source of CP violation", beyond his model with Maskawa. We illustrate this with a simple dimensional analysis of the Jarlskog invariant for CPV, $J \equiv \operatorname{Im} \operatorname{det}\left[m_{u} m_{u}^{\dagger}, m_{d} m_{d}^{\dagger}\right]$ for 3 generations. Expanded, one has

$$
J=\left(m_{t}^{2}-m_{u}^{2}\right)\left(m_{t}^{2}-m_{c}^{2}\right)\left(m_{c}^{2}-m_{u}^{2}\right)\left(m_{b}^{2}-m_{d}^{2}\right)\left(m_{b}^{2}-m_{s}^{2}\right)\left(m_{s}^{2}-m_{d}^{2}\right) A,
$$

where $A \simeq 3 \times 10^{-5}$ is the common triangle area, e.g. for $V_{u d}^{*} V_{u b}+V_{c d}^{*} V_{c b}+V_{t d}^{*} V_{t b}=0$. One has $\mathrm{CPV}$ if and only if $J \neq 0$. Normalizing by electroweak phase transition temperature $T \simeq 100 \mathrm{TeV}$, one finds $J$ seems short by $10^{-10}$ (if not more) to account for BAU.

As a byproduct of "Nature writing" [6] (explaining CPV "to biologists"), I noticed that if one had 4 generations, then simply shifting "123" in Eq. (3.1) to "234", one finds [16],

$$
J_{(2,3,4)}^{s b}=\left(m_{t^{\prime}}^{2}-m_{c}^{2}\right)\left(m_{t^{\prime}}^{2}-m_{t}^{2}\right)\left(m_{t}^{2}-m_{c}^{2}\right)\left(m_{b^{\prime}}^{2}-m_{s}^{2}\right)\left(m_{b^{\prime}}^{2}-m_{b}^{2}\right)\left(m_{b}^{2}-m_{s}^{2}\right) A_{234}^{s b},
$$

which would be enhanced by $\sim 15$ orders of magnitude over $J$ of Eq. (3.1), brought about by the heavy $t^{\prime}, b^{\prime}$ quark masses, which are taken to be in the range of 300 to $600 \mathrm{GeV}$. The CPV area

\footnotetext{
${ }^{1}$ We remark that the $\mathrm{D} \emptyset$ value of $A_{\mathrm{SL}}$ violates a bound. If it is confirmed in the future, e.g. by LHCb, it probably implies hadronically enhanced $\Delta \Gamma_{s}$ from OPE predictions. See [13]; we do not believe in New Physics enhanced $\Delta \Gamma_{s}$.
} 
factor $A_{234}$ (found phenomenologically to be $\mathscr{O}(10-30)$ ) should not be too different from 1 . A detailed algebraic check showed [17] that indeed Eq. (3.2) is the leading effect, which is due to mass hierarchy mostly. Subleading effects, which are many, are typically at $\sim 1 / 10$. Note that this 1000 trillion enhancement comes from large Yukawa couplings, hence is dynamical.

\section{Cauldron: Large Yukawa and EWSB ?}

The $t^{\prime}$ and $b^{\prime}$ mass bounds are becoming rather heavy at the Tevatron, implying that we are entering the realm of large Yukawa couplings for the search of new heavy chiral fermions.

CDF has pursued $t^{\prime} \rightarrow W q$ search ( $q$ jet flavor unspecified) for several years, now joined by D $\emptyset$ this summer, with [18] $m_{t^{\prime}}<335$ and $296 \mathrm{GeV}$ excluded at 95\% C.L., respectively. However, for both experiments, the observed bounds are somewhat weaker than expected sensitivities. In fact, both experiments show an inkling of excess $(\sim 2 \sigma)$ events at high $H_{T}$ and reconstructed mass. It is not clear whether this is genuine, or common underestimation of QCD backgrounds. CDF has also pursued $b^{\prime} \rightarrow W t$ search [19], where the clean signature of same-sign dileptons give rise to a bound of $338 \mathrm{GeV}$. A more stringent bound is inferred from leptons plus (multi-)jets, but it may be questionable in this case how well one understands QCD background with high number of jets.

In any case, the bounds are now above $300 \mathrm{GeV}$, and one is wary of the so-called "unitarity bound": partial wave unitarity breaks down [20] starting 500-600 GeV! Of course, probability is always conserved, so the breakdown only reflects the fact that strong Yukawa couplings set in, and perturbation theory breaks down. This reminds one further of an old suggestion by Nambu: Could EWSB be due to heavy chiral quarks, $t^{\prime}$ and $b^{\prime}$, above the unitarity bound? I.e., the conjecture that EWSB might be due to $\bar{Q} Q$ condensation $(\langle\bar{Q} Q\rangle \neq 0)$ induced by large Yukawa couplings. To seriously address this, one would need to study Higgs-Yukawa sector on a lattice.

We offer some speculation, keeping an "experimentalist" mindset.

The $\mathrm{SU}(2)_{L} \times \mathrm{U}(1)$ chiral gauge symmetry is experimentally established. Spontaneous symmetry breaking is also experimentally established, with massive $W$ and $Z$ bosons, and massive fermions, too. Since renormalizability (needed for contact with LEP data) depends only on Ward identities, it is unaffected by SSB. One can then take the physical gauge, where no would-be Goldstone bosons, or scalar particles, appear at all. Consider the $g V_{i j} \bar{u}_{i} \gamma_{\mu} L d_{j} W^{\mu}$ gauge coupling vertex, which involves only left-handed quarks. Replacing the $W^{\mu}$ by $k^{\mu} / M_{W} \equiv \hat{k}^{\mu}$ from the longitudinal $W$ propagator, and contract with $\gamma_{\mu}$. Noting that $k=p_{i}-p_{j}$, one finds

$$
g \hat{\not k}_{k} L=g\left(\hat{p}_{i}-\hat{p}_{j}\right) L=g\left(\hat{m}_{i} L-\hat{m}_{j} R\right)=\lambda_{i} L-\lambda_{j} R,
$$

where we use the equation of motion to replace momenta by mass, and in so doing, generated the chirality flip. In the last step, we produce the "Yukawa coupling" of the Goldstone boson — but we started from the gauge coupling! This is not surprising, as the Goldstone particle couples to mass. However, notice we never mentioned the existence of the Higgs particle in our entire discussion.

If a new heavy chiral quark $Q$ exists, can its effective Yukawa coupling generate $\langle\bar{Q} Q\rangle \neq 0$ ?

\section{Prognosis - 2011, 2012, beyond}

On the $\sin 2 \Phi_{B_{s}} \equiv \sin \phi_{s} \equiv-\sin 2 \beta_{s}$ front, there will be a tug-of-war between Tevatron and 
$\mathrm{LHCb}$, depending on its value and how fast LHCb can deliver. But no doubt LHCb would end up the winner. By the way, I will put $4 G$ on the back-burner if $\sin 2 \Phi_{B_{s}}$ turns out to be SM3-like.

As for $t^{\prime} / b^{\prime}$ direct search, things would also turn towards LHC's advantage, once some data is accumulated: bounds would surpass Tevatron's with just $100 \mathrm{pb}^{-1}$ [21]. With $1 \mathrm{fb}^{-1}$, the target for 2011, the expected bound would reach the unitarity bound! Thus, to continue the pursuit beyond 2012, one would need guidance from lattice Higgs-Yukawa studies. We remark that, in the long run (beyond 2020), one could in principle extract CPV phase via the $b^{\prime} \rightarrow s \gamma$ mode [22].

If the 4th generation is discovered at the LHC, one important measurement to fix the flavor/CP parameters would be [9] $K_{L} \rightarrow \pi^{0} v \bar{v}$, which could be done by the KOTO experiment at J-PARC. Though a plethora of measurements can be made, it is not yet clear whether the Super B factories, such as Belle II, could provide definitive measurements. This is a question to be pursued.

\section{References}

[1] W.-S. Hou, R.S. Willey and A. Soni, Phys. Rev. Lett. 58 (1987) 1608.

[2] T. Inami and C.S. Lim, Prog. Theor. Phys. 65 (1981) 297.

[3] A. Arhrib and W.-S. Hou, Eur. Phys. J. C 27 (2003) 555,

[4] B. Holdom, W.-S. Hou, T. Hurth, M.L. Mangano, S. Sultansoy and G. Ünel, PMC Phys. A 3 (2009) 4.

[5] G.D. Kribs, T. Plehn, M.S. Spannowsky and T.M.P. Tait, Phys. Rev. D 76 (2007) 075016.

[6] S.-W. Lin, Y. Unno, W.-S. Hou, P. Chang et al. [Belle collaboration], Nature 452 (2008) 332.

[7] W.-S. Hou, M. Nagashima and A. Soddu, Phys. Rev. Lett. 95 (2005) 141601.

[8] W.-S. Hou, H.-n. Li, S. Mishima and M. Nagashima, Phys. Rev. Lett. 98 (2007) 131801.

[9] W.-S. Hou, M. Nagashima, A. Soddu, Phys. Rev. D 72 (2005) 115007.

[10] D. Hooper, P. Blasi and P. D. Serpico, JCAP 01 (2009) 025.

[11] W.-S. Hou, M. Nagashima and A. Soddu, Phys. Rev. D 76 (2007) 016004.

[12] G. Punzi, PoS EPS-HEP2009 (2009) 022 [arXiv:1001.4886 [hep-ex]].

[13] George W.S. Hou, arXiv:1007.2288 [hep-ph], to appear in proceedings of TOP2010.

[14] W.-S. Hou and C.-Y. Ma, Phys. Rev. D 82 (2010) 036002.

[15] See, e.g. A. Soni, A.K. Alok, A. Giri, R. Mohanta and S. Nandi, Phys. Rev. D 82 (2010) 033009; A.J. Buras, B. Duling, T. Feldmann, T. Heidsieck, C. Promberger and S. Recksiegel, JHEP 1009 (2010) 106; O. Eberhardt, A. Lenz and J. Rohrwild, arXiv:1005.3505 [hep-ph].

[16] W.-S. Hou, Chin. J. Phys. 47 (2009) 134.

[17] W.-S. Hou, Y.-Y. Mao and C.-H.-Shen, Phys. Rev. D 82 (2010) 036005.

[18] Alison Lister, PoS ICHEP2010, this proceedings.

[19] Luca Scodellaro, PoS ICHEP2010, this proceedings.

[20] M.S. Chanowitz, M.A. Furman and I. Hinchliffe, Nucl. Phys. B 153 (1979) 402.

[21] The CMS Collaboration, The CMS physics reach for searches at 7 TeV, CMS NOTE-2010/008.

[22] A. Arhrib and W.-S. Hou, Phys. Rev. D 80 (2009) 076005. 